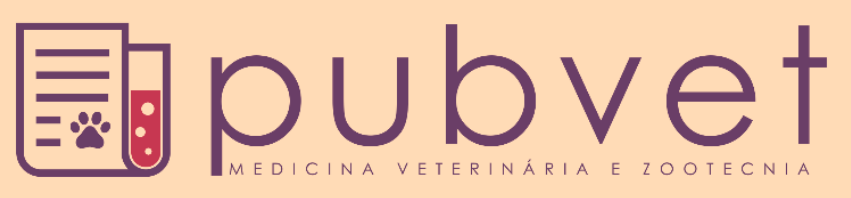

https://doi.org/10.31533/pubvet.v14n8a623.1-10

\title{
Parasitas gastrointestinais em uma criação semi-intensiva de galinhas caipiras, no município de Carmo do Paranaíba, Minas Gerais
}

\author{
Belchior de Oliveira Santana Neto ${ }^{1}$, Nadia Grandi Bombonato ${ }^{2} \theta$, Andresa dos Santos Veras ${ }^{30}$, \\ Renata Lima de Miranda ${ }^{\bullet}$, , Jacqueline Ribeiro de Castro ${ }^{* *}$
}

${ }^{I}$ Graduado em Medicina Veterinária, Centro Universitário de Patos de Minas - UNIPAM - MG, Brasil.

${ }^{2}$ Docente em Medicina Veterinária, Centro Universitário de Patos de Minas - UNIPAM - MG, Brasil

${ }^{3}$ Graduanda em Medicina Veterinária, Faculdade Presidente Antônio Carlos - UNIPAC - MG, Brasil.

${ }^{4}$ Dra. do Programa de Pós-graduação em Imunologia e Parasitologia Aplicada-PIPPA-Universidade Federal de Uberlândia- UFU.

${ }^{5}$ Docente em Medicina Veterinária, Centro Universitário do Triângulo - UNITRI - MG, Brasil.

*Autor para correspondência: E-mail: jack_ufu@yahoo.com.br

Resumo. O frango caipira criado sob os sistemas extensivo e semi-intensivo possui acesso às áreas externas para pastejo e práticas de exercícios, o que permite maior exposição ambiental aos agentes infecciosos. O objetivo do presente estudo foi determinar a presença de parasitas gastrointestinais em um criatório de frangos caipiras, no município de Carmo do Paranaíba, MG, além de estabelecer os possíveis fatores de riscos. O estudo foi realizado em uma criação de 1800 frangos caipiras (Gallus gallus domesticus), criados sob o sistema semi-intensivo de produção, alojados em oito galpões, separadas por fase de criação conforme idade (Pré- inicial 1 e 2- composta por aves de cinco a 10 semanas, Inicial 1 e 210 a 16 semanas e Abate 1 e 2- 16 a 20 semanas). A amostra baseou-se na ocorrência média esperada de 55,4\%, conforme estudo prévio, totalizando 268 amostras, cerca de 45 amostras/box. A determinação da ocorrência de parasitoses foi realizada por meio da colheita de excretas em um papelão posicionado embaixo do poleiro e aguardou-se as aves defecarem e foi colhido cerca de 10 gramas da parte superior das excretas, com posterior avaliação pelos métodos qualitativo (Willis e Hoffman) e quantitativo (n ovos/lâmina). Avaliaram-se os fatores de risco por meio de um questionário epidemiológico, com seleção das variáveis: idade, tipo de alimentação, descarte e mudança periódica da cama dos lotes, controle de entrada e saída das aves nos lotes, vermifugação e vacinação. Utilizou-se um teste não paramétrico Odds Ratio para duas amostras independentes, com um nível de significância de $5 \%$ para análise dos fatores de risco. A ocorrência determinada foi de $57,46 \%(154 / 268)$ e se diferiu entre as fases de criação no sistema semi-intensivo. A fase de abate demonstrou uma menor frequência de ovos $(46,06 \%)$ comparada às fases iniciais (Pré-inicial 61,79\% e Inicial 64,44\%) ( $\mathrm{P}<0,001)$. Foram detectados ovos dos gêneros Ascaridia spp. e Synganus trachea. Dessa forma, concluiu-se que galinhas caipiras pertencentes a uma propriedade rural de Carmo do Paranaíba, MG apresentaram nematoides, sendo as fases iniciais mais parasitadas, ensejando assim, estudos que visem à aplicação de melhores práticas de manejo higiênico sanitárias nesse tipo de criação.

Palavras chave: avicultura, endoparasitoses, frango de corte, nematódeos

\section{Gastrointestinal parasites in a semi-intensive rearing of free-range chickens, in the municipality of Carmo do Paranaíba, Minas Gerais}

Abstract. Free-range chicken raised under extensive and semi-intensive systems has
access to outside areas for grazing and exercise practices, which allows greater
environmental exposure to infectious agents. The goal of the present study was to
determine the presence of gastrointestinal parasites in a free-range chicken farm in the
municipality of Carmo do Paranaíba, MG, in addition to establishing the possible risk 
factors. The study was carried out in a breeding of 1800 free-range chickens (Gallus gallus domesticus), bred under the semi-intensive production system, housed in eight sheds, separated by breeding phase according to age (Phases: Pre-initial 1 and 2- compound by birds of five to 10 weeks, Initial 1 and 2- 10 to 16 weeks and Slaughter 1 and 2-16 to 20 weeks). The sample was based on the expected average occurrence of $55.4 \%$, according to a previous study, totaling 268 samples, about 45 samples / box. The determination of the occurrence of parasites was carried out by collecting excreta in a cardboard positioned under the perch and the birds were expected to defecate and about 10 grams of the upper part of the excreta were collected, with subsequent evaluation by qualitative (Willis and Hoffman) and quantitative methods (n eggs/blade). Risk factors were assessed using an epidemiological questionnaire, with selection of the variables: age, type of feeding, disposal and periodic change of flock litter, control of entry and exit of birds in the flocks, deworming and vaccination. A non-parametric Odds Ratio test was used for two independent samples, with a significance level of $5 \%$ for the analysis of risk factors. The determined occurrence was $57.46 \%(154 / 268)$ and differed between the breeding phases in the semi-intensive system. The slaughter phase showed a lower frequency of eggs (46.06\%) compared to the initial phases (Pre-initial 61.79\% and Initial 64.44\%) (P <0.001). Eggs of the genera Ascaridia spp. and Synganus trachea. Thus, it was concluded that free-range chickens belonging to a rural property in Carmo do Paranaíba, MG had nematodes, the initial phases being more parasitized, thus giving rise to studies aimed at applying better sanitary hygiene management practices in this type of breeding.

Keywords: poultry, endoparasitoses, broiler chicken, nematodes

\section{Parásitos gastrointestinales en un sistema semiintensivo de pollos de corral, en el municipio de Carmo do Paranaíba, Minas Gerais}

Resumen. El pollo de corral criado bajo sistemas extensivos y semi-intensivos tiene acceso a áreas externas para prácticas de pastoreo y ejercicio, lo que permite una mayor exposición ambiental a agentes infecciosos. El objetivo del presente estudio fue determinar la presencia de parasitas gastrointestinales en una granja de pollos en el municipio de Carmo do Paranaíba, MG, además de establecer los posibles factores de riesgo. El estudio se llevó a cabo en una producción de 1800 pollos de corral (Gallus gallus domesticus), criados bajo el sistema de producción semintensivo, alojados en ocho cobertizos, separados por fase de cría según la edad (Pre-inicial 1 y 2: compuesto por aves de cinco a 10 semanas; inicial 1 y 2: 10 a 16 semanas; sacrificio 1 y 2:16 a 20 semanas). La muestra se basó en la casuística promedio esperada del 55.4\%, según un estudio anterior, totalizando 268 muestras, alrededor de 45 muestras/caja. La determinación de la aparición de parásitos se realizó mediante la recolección de excretas en un cartón colocado debajo de la percha y se esperó que las aves defecaran y se recolectaron aproximadamente 10 gramos de la parte superior de las excretas, con una evaluación posterior por métodos cualitativos (Willis y Hoffman) y cuantitativo (n huevos/lámina). Los factores de riesgo se evaluaron mediante un cuestionario epidemiológico, con selección de las variables: edad, tipo de alimentación, eliminación y cambio periódico de la cama de los lotes, control de entrada y salida de aves en los lotes, vermifugación y vacunación. Se utilizó una prueba no paramétrica Odds Ratio para dos muestras independientes, con un nivel de significancia del 5\% para el análisis de los factores de riesgo. La casuística determinada fue $57.46 \%(154 / 268)$ y difirió entre las fases de reproducción en el sistema semiintensivo. La fase de sacrifício mostró una menor frecuencia de huevos (46.06\%) en comparación con las fases iniciales (Pre-iniciale $61.79 \%$ e Inicial de 64.44\%) (P <0.001). Huevos de los géneros Ascaridia spp. y Synganus trachea $a$ fueron detectados. Por lo tanto, se concluyó que los pollos de corral pertenecientes a una propiedad rural en Carmo do Paranaíba, MG, tenían nematodos, las fases iniciales estaban más parasitadas, lo que dio lugar a estudios destinados a aplicar mejores prácticas de gestión de higiene sanitaria en este tipo de cría.

Palabras clave: avicultura, endoparasitosis, pollo de engorde, nematodos 


\section{Introdução}

O frango caipira, também denominado frango colonial, criado sob os sistemas extensivo e semiintensivo possui acesso às áreas externas para pastejo, práticas de exercícios e com manifestação de comportamento inerente à espécie. Alimenta-se com ração constituída por ingredientes preferencialmente de origem vegetal, sem a aplicação de substâncias para a melhoria de desempenho (Siqueira \& Marques, 2016). Esse tipo de criação permite maior exposição ambiental dessas aves aos agentes infecciosos, como os causadores de enfermidades parasitárias, as quais representam causa frequente de redução de índices reprodutivos e produtivos do plantel afetado. As parasitoses intestinais encontram-se difundidas na criação extensiva na avicultura com relevante morbimortalidade. Aves infectadas podem apresentar atraso no crescimento, redução na conversão alimentar e elevação da suscetibilidade para outros agentes infecciosos (Cardozo \& Yamamura, 2004; Lozano et al., 2019; Rennó et al., 2008).

Clinicamente, as aves acometidas por enteroparasitoses podem apresentar apatia, prostração, hiporexia, perda de peso, síndrome da má absorção, anemia, aumento de volume abdominal, diarreia e manifestações neurológicas. Algumas infecções parasitárias facilitam a entrada de bactérias, vírus e fungos, condição na qual, favorece a instalação de comorbidades (Siqueira \& Marques, 2016).

As endoparasitoses caracterizam-se por ser causadas por agentes que parasitam internamente seus hospedeiros (Rennó et al., 2008). De acordo com Siqueira \& Marques (2016) há uma variedade de helmintos que parasitam as aves, sendo os nematóides e os cestóides, mais ocorrentes nessa espécie. No Brasil, já foram identificados 17 gêneros diferentes de nematóides em galinhas domésticas (Vicente et al., 1995), sendo os mais frequentes gêneros Ascaridia, Capillaria, Heterakis e Strongyloides (Boroviec et al., 2020; Carneiro, 2001; Cazanti et al., 2007; Fernandes et al., 2005; Gomes et al., 2009; Vieira, $\underline{2010})$.

Além das parasitoses verminóticas que podem causar perdas consideráveis na avicultura mundial, destacam-se as coccidioses. Costa (2002) e Williams (1999) enfatizaram que a ocorrência do coccídeo do gênero Eimeria, cuja presença é relatada em parte majoritária dos levantamentos coproparasitológico na avicultura brasileira.

Acredita-se que a aplicação de métodos laboratoriais baseados na pesquisa e identificação de ovos e oocistos nas fezes de aves domésticas poderão contribuir no estabelecimento do controle estratégico das endoparasitoses em criações de aves, com elevações significativas dos índices produtivos desse segmento, assim como foi observado em estudo realizado por Gomes et al. (2009).

Dessa forma, objetivou-se determinar a presença de parasitas gastrointestinais em criatório semiintensivo de galinhas caipiras, no município de Carmo do Paranaíba, MG, além de estabelecer os possíveis fatores de riscos envolvidos na ocorrência das parasitoses gastrointestinais.

\section{Material e métodos}

Realizou-se o estudo em conformidade as normas estabelecidas pelo Comitê de Ética do uso de Animais (CEUA) do Centro Universitário de Patos de Minas (UNIPAM) (Parecer favorável: 119/17) e após prévia autorização dos responsáveis.

\section{Local do estudo}

Um estudo observacional transversal prospectivo foi realizado em uma criação de frangos/galinhas caipiras (Gallus gallus domesticus, Linnaeus, 1758), localizada no perímetro rural no município de Carmo do Paranaíba, Minas Gerais, Brasil 354, km 269, situada na mesorregião do Triângulo Mineiro/Alto Paranaíba com municípios limítrofes Patos de Minas, Rio Paranaíba, Tiros, Lagoa Formosa, Serra do Salitre e Arapuá (Figura 1). O município localiza-se a uma altitude de $1061 \mathrm{~m}$, clima tropical e a economia do município relaciona-se diretamente às atividades agropecuárias, que constituem seu setor mais dinâmico (Wikipedia, 2017). 


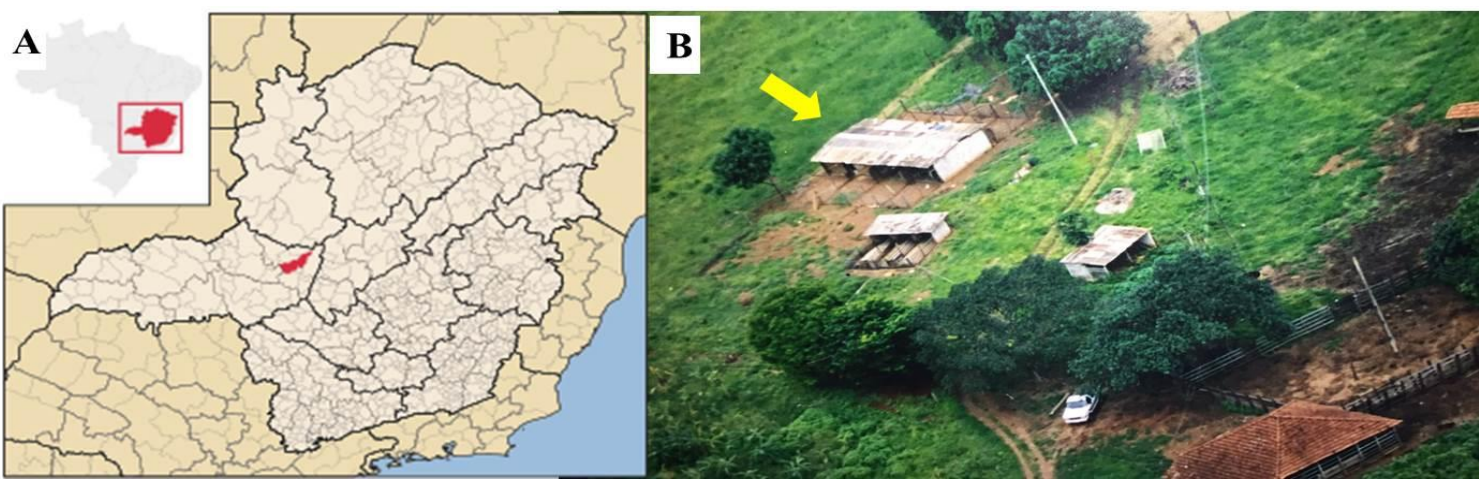

Figura 1. Localização da granja no município de Carmo do Paranaíba, no estado de Minas Gerais (A). Galpões da granja (seta) de galinhas caipiras em sistema semi-intensivo de produção, Carmo do Paranaíba, MG, 2017. Fonte: Wikipedia, 2017. B- Arquivo pessoal.

\section{População estudada e delineamento amostral}

Foi avaliada uma granja de galinhas caipiras criadas sob o sistema semi-intensivo de produção, alojadas conforme o esquema o esquema 1. A propriedade é constituída de aproximadamente $1500 \mathrm{a}$ 1800 aves cruzadas compostas por pintainhos, frangos e galinhas. No momento do estudo, haviam 1800 aves alojadas. Constituída por oito galpões (box) com área de cobertura e área livre extensiva totalizando em 7,5 m²/box. Com lotação média de alojamento de 40 aves $/ \mathrm{m}^{2}$. Notou-se que o manejo sanitário no box um e oito se diferem dos demais por estarem em vazio sanitário, não ocupados, o tempo e ocupação de cada box dois a três meses conforme idade e peso aproximados para uniformização dos lotes.

Esquema 1. Esquema espacial da granja de galinha caipira em sistema semi-intensivo de produção que foi estudada para a determinação da presença de parasitas gastrointestinais, Carmo do Paranaíba, MG, 2017.

\begin{tabular}{|c|c|c|c|}
\hline Box 1 & Box 2 & Box 3 & Box 4 \\
\hline $\begin{array}{c}\text { Solo } \\
4 \mathrm{~m}^{2} \\
\text { Vazio Sanitário }\end{array}$ & $\begin{array}{l}\text { Solo } \\
4 \mathrm{~m}^{2}\end{array}$ & $\begin{array}{l}\text { Solo } \\
4 \mathrm{~m}^{2}\end{array}$ & $\begin{array}{l}\text { Solo } \\
4 \mathrm{~m}^{2}\end{array}$ \\
\hline $\begin{array}{c}\text { Área coberta e } \\
\text { Cimentada } \\
3,5 \mathrm{~m}^{2}\end{array}$ & $\begin{array}{c}\text { Área coberta e } \\
\text { Cimentada } \\
3,5 \mathrm{~m}^{2}\end{array}$ & $\begin{array}{c}\text { Área coberta e } \\
\text { Cimentada } \\
3,5 \mathrm{~m}^{2}\end{array}$ & $\begin{array}{c}\text { Área coberta e } \\
\text { Cimentada } \\
3,5 \mathrm{~m}^{2}\end{array}$ \\
\hline $\begin{array}{c}\text { Área coberta e } \\
\text { Cimentada } \\
3,5 \mathrm{~m}^{2} \\
\end{array}$ & $\begin{array}{c}\text { Área coberta e } \\
\text { Cimentada } \\
3,5 \mathrm{~m}^{2}\end{array}$ & $\begin{array}{c}\text { Área coberta e } \\
\text { Cimentada } \\
3,5 \mathrm{~m}^{2}\end{array}$ & $\begin{array}{c}\text { Área coberta e } \\
\text { Cimentada } \\
3,5 \mathrm{~m}^{2} /\end{array}$ \\
\hline $\begin{array}{l}\text { Solo } \\
4 \mathrm{~m}^{2}\end{array}$ & $\begin{array}{l}\text { Solo } \\
4 \mathrm{~m}^{2}\end{array}$ & $\begin{array}{l}\text { Solo } \\
4 \mathrm{~m}^{2}\end{array}$ & $\begin{array}{c}\text { Solo } \\
4 \mathrm{~m}^{2} \\
\text { Vazio Sanitário }\end{array}$ \\
\hline
\end{tabular}

A criação estava aproximadamente há seis meses sem receber vermifugação o que possibilitou a realização do estudo sem interferência de fator residual conferido pela medicação.

O delineamento amostral foi baseado na prevalência média esperada de 55,4\% conforme estudo previamente realizado por (Siqueira \& Marques, 2016), totalizando 268 amostras. Mediante a distribuição das aves na propriedade foi calculado cerca de 45 amostras/Box (Esquema 2).

A colheita do material foi padronizada entre os lotes ocupados, exceto no box dos pintainhos, que devido à pequena quantidade de fezes, em gramas, durante acolheita resultou em amostra insatisfatória e foram retirados do estudo.

Os galpões avaliados estavam ocupados por aves pertencentes às fases pré- inicial 1, pré-inicial 2 , inicial 1, inicial 2 e abate 1 e 2 . As idades dos lotes foram estimadas, pois, tratava-se de frangos caipiras, os quais não apresentam padronização durante o nascimento e por serem adquiridos de diferentes propriedades, sem uniformidade, devido cruzamentos desprovidos de padrão racial. A realocação em 
nova fase nessa granja é feita pela observação do crescimento ponderal, peso e perfil racial predominante no cruzamento.

Esquema 2. Demonstração do cálculo amostral do estudo na determinação da presença de parasitas gastrointestinais de galinha caipira em sistema semi-intensivo de produção de uma granja comercial, Carmo do Paranaíba, MG, 2017.

$n=\frac{\mathbf{Z}^{2} \mathbf{a} / 2 \cdot \mathbf{p} \cdot \mathbf{q}}{\mathrm{e}^{2} \mathbf{a} /^{2}}$

Substituindo os valores na fórmula:

$n=\frac{1,962.0,554.0,446}{(0,0548)^{2}}$

$\mathbf{n}=\underline{\mathbf{3 1 6} \text { aves }}$

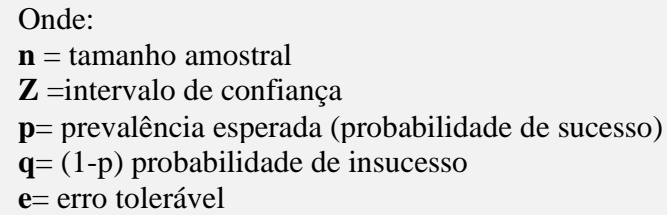

O número de amostras/box conforme fases encontra-se detalhada a seguir: fase pré-inicial $1=45$; pré-inicial $2=44$; inicial $1=45$; inicial $2=45$; abate $1=45$; abate $2=44$ aves. As idades dos lotes foram: fase pré-inicial composta por aves de cinco a 10 semanas, a fase inicial constituída por aves de 10 a 16 semanas e a fase final de abate aves com 16 a 20 semanas.

\section{Coleta das excretas}

As amostras de excretas foram colhidas e identificadas conforme o lote de origem. O procedimento de colheita foi por meio de um papelão posicionado embaixo do poleiro e aguardava-se a ave defecar. Colhia-se apenas a parte superior das excretas desprezando a parte inferior que entrou em contato direto com o papelão, conforme o protocolo determinado por Batista et al. (2008) e Hoffman (1987) que preconizam que a colheita de fezes deve ser feita retirando apenas as partes superiores e internas das amostras, evitando assim, a coleta de materiais com sujidades.

A colheita de 10 gramas de excreta/ave foi realizada com auxílio de uma espátula individual e foram acondicionadas em embalagens plásticas identificadas e foram mantidas sob refrigeração, com auxílio de uma caixa com gelo para transporte. Após as amostras foram mantidas na geladeira $\left(4\right.$ a $\left.8^{\circ} \mathrm{C}\right)$ por até 48 horas e após encaminhadas para o Laboratório de Parasitologia do Centro Universitário de Patos de Minas (UNIPAM) para o processamento do exame coproparasitológico.

\section{Análise laboratorial}

Cada amostra foi submetida aos métodos de análise qualitativa de Willis (1921) (flutuação com solução saturada de cloreto de sódio) conforme Quadros et al. (2015), sedimentação simples espontânea (Hoffmann, 1987; Neves et al., 2005) e análise quantitativa.

Na técnica de Willis (1921), pesaram-se 2 gramas de amostra fecal na balança analítica (Figura 2A) e foi acondicionada em copo transparente descartável de plástico. Após acresceu solução saturada $\mathrm{NaCl}$ $35,9 \%$ para homogeneizar as fezes, com auxílio de um bastão de vidro (Figura 2B). A amostra foi filtrada em uma peneira com uma gaze dobrada em quatro partes posicionada na peneira (Figura 2C). Após colocou-se a amostra filtrada em um tubo de ensaio até formar o menisco positivo excedendo o bordo superior do tubo e foi colocada uma lamínula de vidro por 15 minutos sobre o tubo de ensaio (Figura 2D). Retirou-se a lamínula e inverteu a rapidamente sem deixar cair a gota de solução e foi aderida em uma lâmina de vidro. Avaliou sob microscopia óptica (Figura 2F), na objetiva de 100x, com uma gota de Lugol (Moreira et al., 2017).

Na técnica de Hoffman (1987), pesaram-se 2 gramas de amostra e em seguida, realizou-se a dissolução e homogeneização das fezes utilizando água $(100 \mathrm{~mL})$. A filtragem da amostra diluída foi feita em peneira e acondicionada direto no cálice de vidro (Figura 2E) e completou-se com água até atingir um volume de $125 \mathrm{~mL}$. Deixou-se em repouso por 20 minutos e realizou-se a troca do sobrenadante turvo de forma cuidadosa, a fim de que, não ocorresse perda do sedimento acumulado no 
fundo do cálice, colocando $125 \mathrm{~mL}$ novamente. Após a primeira troca do sobrenadante, realizou-se mais uma, respeitando os 20 minutos, até que o mesmo ficasse translúcido, com o sedimento ao fundo do frasco e finalmente eliminou-se o sobrenadante presente no cálice para facilitar a sucção pela pipeta de Pasteur do sedimento. Colocou-se uma gota dos sedimentos na lâmina, e com outra pipeta, pôs-se uma gota de Lugol e sobrepôs uma lamínula para ser analisada ao microscópio óptico com a lente de aumento de 10x (Moreira et al., 2017).

Para análise quantitativa dos ovos encontrados foi realizada a varredura completa da lâmina em microscopia óptica em aumento de no aumento de 10x. A classificação de infecção endoparasitária adotada no presente estudo foi conforme os critérios do estudo de Hoffmann (1987) que dispôs a intensidade do parasitismo de acordo com o número de ovos encontrados por lâminas analisadas, sendo a classificada como raríssimo (1-3 ovos/análise), raro (4-5ovos/análise), pequena quantidade (6-10), quantidade regular (11-20 ovos/análise), grande quantidade (21-50 ovos/análise) e extraordinária quantidade ( $\geq 51$ ovos/análise).
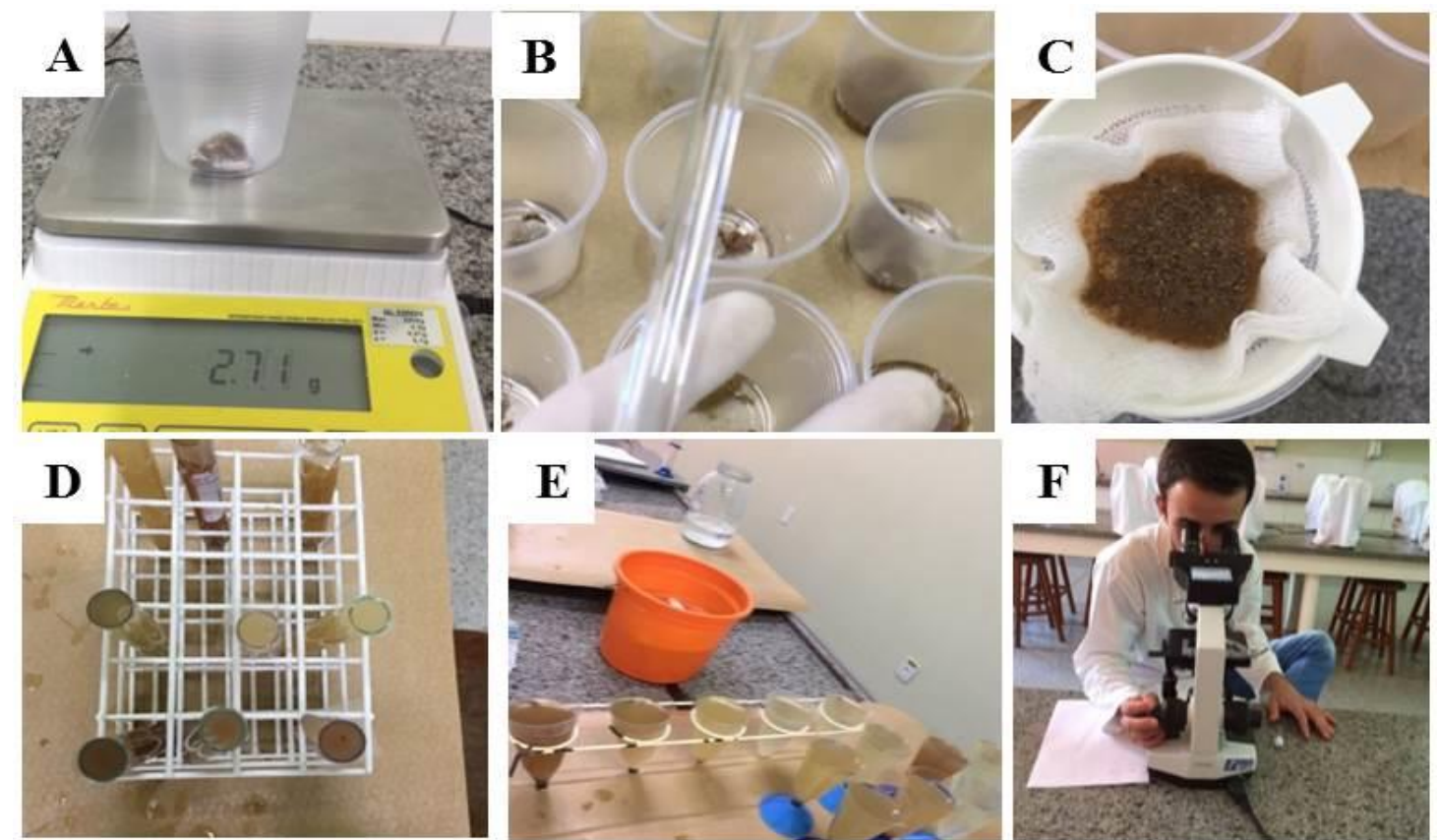

Figura 2. Etapas do exame coproparasitológico para a determinação da presença de parasitas gastrointestinais de galinhas caipiras de uma granja comercial do Carmo do Paranaíba, MG, 2017. A- Pesagem das amostras de excretas; B- Diluição e homogeneização das amostras em solução saturada $\mathrm{NaCl}$ para técnica de Willis; CFiltração das amostras em peneira e gaze; D- Amostras filtrada em um tubo de ensaio até formar o menisco positivo com a colocação de lamínulas na técnica de Willis; E- Amostra acondicionadas nos cálices de vidro em repouso para análise do sedimento na técnica de Hoffman; F- Leitura das amostras em microscópio óptico. Fonte: Arquivo pessoal. Análises realizadas no Laboratório de Parasitologia do Centro Universitário de Patos de Minas (UNIPAM, MG).

\section{Questionário epidemiológico e manejo sanitário}

Foi confeccionado um questionário epidemiológico para investigação dos possíveis fatores de risco envolvidos na ocorrência das endoparasitoses da referida propriedade. Perguntas fechadas, objetivas e dicotômicas, com respostas do tipo binária foram elaboradas. Alguns questionamentos foram confeccionados com múltipla escolha e destinado espaço para possíveis comentários (Vieira, 2009). Realizaram-se indagações ao proprietário em relação ao controle de entrada e saída das aves, fonte de agua e se é tratada, se é feito o descarte da cama do aviário, qual tipo de cama e usada, protocolo de vermifugação e vacinação das aves, periodicidade que o mesmo é realizado, tipo de bebedouro usado na propriedade, acesso a pastejo dos galpões e vazio sanitário.

De modo sumarizado o manejo sanitário da granja era baseado na manutenção de rodízio entre os lotes e vazio sanitário de no mínimo 20 dias até a chegada de um novo lote. As aves eram imunizadas contra as doenças de New Castle, Gumboro e Bouba Aviaria. Vermifugadas com mebendazol 
trimestralmente. As aves eram manejadas em lotes e recebem alimentação conforme cada fase. Aves até os 30 dias foram suplementadas com ração para crescimento (Agroceres ${ }^{\circledR}$ ), após suplementadas com milho moído e concentrado elaborado para a fase inicial, recebendo também pastagem e milho em grão. O tempo de alojamento médio para das aves na propriedade é cerca de quatro meses até o abate. As aves foram comercializadas em São Gotardo e Carmo do Paranaíba, MG.

\section{Análise estatística}

Para cálculo da frequência foi estabelecido relação do número de animais positivos no exame coproparasitológico pelo número de animais amostrados, utilizando-se análise estatística descritiva por meio de frequência absoluta e relativa.

Utilizou-se um teste não paramétrico Odds Ratio para duas amostras independentes, para determinação de fatores de risco, com um nível de significância de 5\%. As variáveis foram submetidas ao teste de Shapiro-Wilk como teste de distribuição de normalidade $(\mathrm{P}>0,05)$. A presença de parasitas nas fases de criação foi comparada pelo teste de Kruskal-Wallis, para variáveis não paramétricas (comparação entre três ou mais grupos) e caso detectada diferença significativa $(\mathrm{p}<0,05)$ o pós-teste de Dunn foi selecionado. O programa utilizado para a análise estatística foi o Graphpad versão 5.0 para Windows (San Diego, Califórnia, USA).

\section{Resultados e discussão}

A presença de parasitas gastrointestinais em um criatório semi-intensivo de galinhas caipiras, no município de Carmo do Paranaíba, MG foi de 57,46\% (154/268). A ocorrência de parasitas gastrointestinais em galinhas caipiras se diferiu entre as fases de criação no sistema semi-intensivo avaliado $(\mathrm{P}<0,001)$. A maior ocorrência de ovos no coproparasitológico foi identificada nas fases préinicial 1 e inicial 1 (com $80 \%$ dos animais acometidos), seguida pelo abate 1 (50\%), de acordo com a tabela 1. Esse fato pode estar relacionado pela realocação das aves, novos animais provindos de outros locais (compra externa) e a mudança do manejo nutricional com alteração na composição do concentrado inicial nas fases pré-iniciais, concentrado de crescimento nas fases iniciais e sem concentrado na fase de abate (ração nessa fase de abate composta por e milho moído, farelo de soja e milho em grão, além da pastagem presente em todas as fases).

Tabela 1. Presença de parasitas gastrointestinais em granja de galinha caipira em sistema semi-intensivo de produção, Carmo do Paranaíba, MG, 2017.

\begin{tabular}{|c|c|c|c|c|c|c|c|}
\hline Fases & $\mathrm{N}$ & $\mathrm{n}$ & Amostras positivas & $\begin{array}{l}\text { Amostras } \\
\text { negativas }\end{array}$ & $\begin{array}{c}\text { Ascaridia } \\
\text { (n ovos) }\end{array}$ & $\begin{array}{c}\text { Synganus } \\
\text { (n ovos) }\end{array}$ & $\begin{array}{c}\text { Ocorrência } \\
(\%)\end{array}$ \\
\hline Pré-Inicial 1 & 300 & 45 & 36 & 9 & 64 & 62 & 80 \\
\hline Pré- Inicial 2 & 300 & 44 & 19 & 25 & 33 & 35 & 43 \\
\hline Inicial 1 & 300 & 45 & 36 & 9 & 34 & 35 & 80 \\
\hline Inicial 2 & 300 & 45 & 22 & 23 & 38 & 42 & 49 \\
\hline Abate 1 & 300 & 44 & 22 & 22 & 39 & 34 & 50 \\
\hline Abate 2 & 300 & 45 & 19 & 26 & 36 & 32 & 42 \\
\hline Total & 1800 & 268 & 154 & 114 & 244 & 240 & 57,46 \\
\hline
\end{tabular}

$\mathrm{N}=$ número total de aves em cada box; $\mathrm{n}=$ número de animais amostrados no estudo.

Galha et al. (2008) apontam o estresse e o manejo alimentar como principais causas de imunossupressão em aves, podendo aumentar sua suscetibilidade a doenças infecciosas com enteroparasitoses. Em aves criadas comercialmente, o estresse pode ser induzido por diversos fatores como temperatura ambiente, ventilação, qualidade do ar, densidade populacional, competição por alimento e água de beber, nível de ruído no ambiente e fotoperíodo em que as aves são submetidas.

A frequência encontrada no presente estudo foi semelhante ao estudo de Siqueira \& Marques (2016) que avaliaram 13 propriedades e determinaram uma prevalência geral de 55,4\% (62/112) de aves parasitadas. Embora a ocorrência detectada ser relevante, o criador não evidenciou nenhum problema sanitário nas aves, o que pode ser explicado possivelmente pela baixo índice de parasitas/aves 
detectados, com mais da metade das aves avaliadas com nematoides, os animais positivos apresentaram aproximadamente em média três ovos (total de ovos identificados $=484 / \mathrm{n}$ aves positivas $=154$ ), sendo considerada uma parasitose baixa e raríssima, conforme o padrão de Hoffmann (1987), sem impacto produtivo. Já Brandão et al. (2008) e Puttalakshmamma et al. (2008) que avaliaram também galinhas caipiras encontraram índices maiores de parasitas gastrointestinais, com ocorrências de 91,40\% e 68,1\%, respectivamente. A elevada frequência de enteroparasitoses foi explicada pela falta de vermifugação no sistema extensivo avaliado, devido ao criador não considerar necessária a vermifugação devido à percepção de que as galinhas são saudáveis, com raros casos de óbito. Embora parasitadas, as aves apresentam resistência, provavelmente pelo fato de estarem soltas, isentas de stress ou apresentarem um grau de infecção baixo.

Foram detectados ovos dos gêneros Ascaridia spp. e Synganus trachea, localizados no intestino delgado e traqueia das aves, respectivamente (Figura 3) corroborando com a literatura que destaca os nematoideos dos gêneros mais frequentes em aves domésticas sendo Ascaridia, Capillaria, Heterakise Strongyloides (Brandão et al., 2008; Carneiro, 2001; Fernandes et al., 2005; Gomes et al., 2009; Lima et al., 2011; Lozano et al,, 2019; Vieira, 2010).

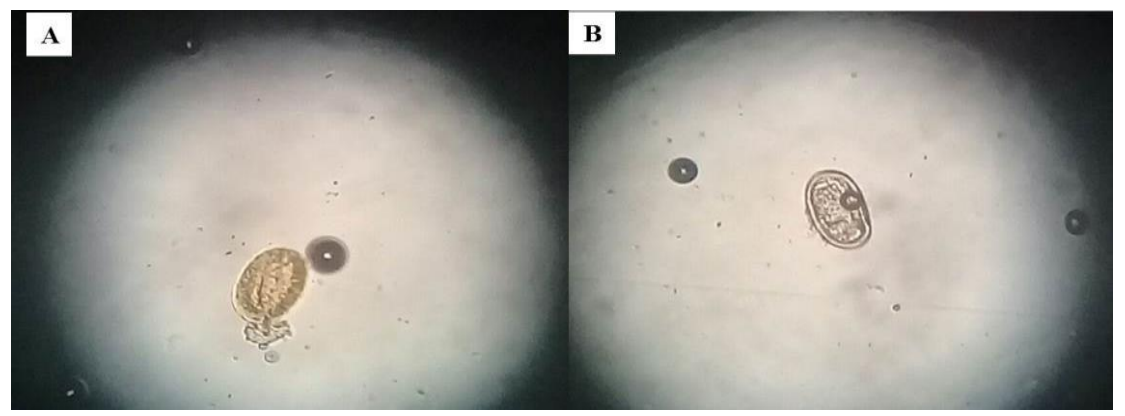

Figura 3. Identificação de ovos dos gêneros Ascaridia spp. (A) e Synganus trachea (B) em galinhas caipiras do Carmo do Paranaíba, MG, 2017

O presente estudo difere de Carneiro (2001) que também avaliou galinhas caipiras e identificou uma maior diversidade de enteroparasitas, sendo oito espécies de helmintos: Amoebo taeniacuneata, Oxyspirura mansoni, Gongylonemaingluvicola, Davainea proglotina, Raillietina echinobothrida, Raillietina tetragona, Tetrameres confusa, Cheilospirurahamulosa. Uma possível explicação por não ter sido identificado cestoideo e uma menor variedade de ovos, talvez pelo fato, da base da vermifugação ser mebendazol, mesmo estando em atraso, conforme informado no inquérito epidemiológico pelo produtor, ainda ter efeito residual e pela pressão ambiental distinta, possivelmente associada a condições de resistência e imunidade das aves avaliadas.

Não foram detectados coccidiose diferindo de estudos que apontam que a eimeriose afeta comumente frangos de corte, galinhas poedeiras e matrizes e que a coccidiose aviária é considerada como uma das mais importantes doenças na indústria avícola (Galha et al., 2008; Prakashbabu et al., 2017; Siqueira \& Marques, 2016). Esse fato pode estar relacionado ao tipo de manejo adotado em um sistema semiintensivo, com uma lotação bem menor que o sistema de criação intensivo, reduzindo os fatores de risco associados, como competição alimentar.

Apenas nematódeos foram encontrados na presente pesquisa. Siqueira \& Marques (2016) identificaram ovos de helmintos dos gêneros Capillaria, Heterakis, Ascaridia, Strongyloides, Strongyloidea e protozoários do gênero Eimeria, com o único cestóide encontrado foi o do gênero Choanotaenia. Capillaria spp. Foi helminto mais prevalente $(78,6 \%)$ independente de município estudado nesse estudo de Siqueira \& Marques (2016). Synganus são mais comuns em galináceos jovens, sendo também descritos em patos, gansos e pássaros silvestres. Rennó et al. (2008) determinaram uma frequência de 3,3\% de Syngamus trachea em galinhas caipiras em pequenas propriedades da região serrana de Santa Catarina.

Avaliou-se ainda a presença de parasitas nas galinhas (Tabela 2) agrupadas conforme a idade considerando como critério aves jovens ( $\leq 10$ semanas) e adultas ( $>10$ semanas) e de acordo com a fase de alojamento (pré-inicial, inicial e abate). A fase de abate demonstrou uma menor frequência de ovos 
de nematoides comparada às fases iniciais, devido um maior tempo e adaptação ao ambiente, exposição e desenvolvimento de imunidade comparada às aves mais jovens alojadas nas fases iniciais (Galha et al., 2008).

Conforme, Boroviec et al. (2020), as parasitoses intestinais afetam principalmente aves jovens, enquanto que, aves com mais de algumas semanas de vida tornam-se em geral portadoras intestinais assintomáticas

Tabela 2. Distribuição das aves parasitadas de acordo com a idade e a fase de criação em granja de galinha caipira em sistema semi-intensivo de produção, Carmo do Paranaíba, MG, 2017.

\begin{tabular}{|c|c|c|c|c|c|c|}
\hline \multirow{2}{*}{ Variáveis } & \multicolumn{3}{|c|}{ Aves } & \multirow{2}{*}{$O R^{*}$} & \multirow{2}{*}{ IC $95 \%$} & \multirow{2}{*}{$\mathrm{P}$} \\
\hline & Total & Positivas & Frequência (\%) & & & \\
\hline \multicolumn{7}{|l|}{ Idade } \\
\hline Jovens (até 10 semanas) & 89 & 55 & 61,79 & \multirow{2}{*}{1,45} & \multirow{2}{*}{$0,86-2,43$} & \multirow{2}{*}{0,15} \\
\hline Adultas (> 10 semanas) & 179 & 99 & 55,30 & & & \\
\hline \multicolumn{7}{|l|}{ Fases } \\
\hline Pré-inicial & 89 & 55 & 61,79 & \multirow{3}{*}{$0,49 * *$} & \multirow{3}{*}{$0,29-0,83$} & \multirow{3}{*}{0,0089} \\
\hline Inicial & 90 & 58 & 64,44 & & & \\
\hline Abate & 89 & 41 & 46,06 & & & \\
\hline
\end{tabular}

*Odds Ratio; **OR realizado para a maior diferença entre as proporções; $\mathrm{p}<0,05$ demonstra diferença significativa.

\section{Conclusão}

Conclui-se que as galinhas caipiras da referida propriedade apresentaram nematoides dos gêneros Ascaridia spp. e Synganus trachea e que, a fase de abate demonstrou ser menos acometida por de parasitas gastrointestinais quando comparada às fases iniciais, ensejando assim, estudos que visem à aplicação de melhores práticas de manejo higiênico sanitárias nesse tipo de criação.

\section{Referências bibliográficas}

Batista, A. M. B., Pereira, M., \& Vita, G. F. (2008). Intestinal parasitism on ostriches (Struthio camelus australis Linnaeus, 1786) from South Region of Espírito Santo State, in 2006. Revista Portuguesa de Ciências Veterinárias, 103(1), 189-193.

Boroviec, B. B., Gasparotto, P. H. G., Dantas Filho, J. V., Peixoto, R. M., Viana, G. A., Rocha, A. S. C. M., Daudt, C., \& Silva, F. R. C. (2020). Occurrence of Ascaridia galli and Heterakis gallinarum in Guinea Fowl (Numida meleagris) in the State of Rondônia, Brazil. Acta Scientiae Veterinariae, 48, 487. https://doi.org/10.22456/1679-9216.100099

Brandão, P. A., Sobral, E. S., Brito, I. C. A., Silva, S. G., Silva, I. K. C., \& Costa, V. M. M. (2008). Prevalência de endoparasitoses em galinha caipira em assentamento rural no semi-árido paraibano. Anais 5o Congresso Nordestino de Produção Animal.

Cardozo, S. P., \& Yamamura, M. H. (2004). Parasitas em produção de frangos no sistema de criação tipo colonial/caipira no Brasil. Semina Ciências Agrarias, 25(1), 63-74.

Carneiro, V. (2001). Composição e estrutura da comunidade de helmintos parasitos de galinhas, Gallus domesticus (L.), no Município de Seropédica, Estado do Rio de Janeiro. 2001. Tese (Doutorado) Universidade Federal Rural do Rio de Janeiro, RJ.

Cazanti, D., Carraro, M., Jussara, M., \& Leonardo, L. O. (2007). Perfil parasitológico de aves de produção. V Encontro Internacional de Produção Científica-Cesumar, 1.

Costa, C. A. F. (2002). Controle da coccidiose: possíveis avanços. Simpósio de Sanidade Avícola Da UFSM, 3, 36-47.

Fernandes, R. M., Rodrigues, M. L. A., Borba, H. R., Fernandes, M., \& Amorim, A. (2005). Atividade anti-helmíntica de plantas em frangos de corte naturalmente infectados com Ascaridia galli. Arquivo Brasileiro de Medicina Veterinária e Zootecnia, 57, 264-266. https://doi.org/10.1590/s0102$\underline{09352005000800020}$

Galha, V., Bondan, E. F., \& Lallo, M. A. (2008). Relação entre imunossupressão e coccidiose clínica em frangos de corte criados comercialmente* Relationship between immunosuppression and clinical 
coccidiosis in broiler chicks commercially raised. Revista Do Instituto de Ciência Da Saúde, 26(4), 432-437.

Gomes, F. F., Simões Machado, H. H., Lemos, L. S., Almeida, L. G., \& Daher, R. F. (2009). Principais parasitos intestinais diagnosticados em galinhas domésticas criadas em regime extensivo na municipalidade de Campos dos Goytacazes, RJ. Ciência Animal Brasileira, 10(3), 818-822.

Hoffmann, R. P. (1987). Diagnóstico de parasitismo veterinário. Sulina.

Lima, E. M., Santos, M. S. V., Tavares, F. B., Andrade, P. A., Costa, H. S. (2011). Perfil parasitológico intestinal de frangos caipiras criados em diferentes sistemas de criação. Anais 9o Seminário Anual de Iniciação Científica, Parapuapebas, AM, (Resumo 72).

Lozano, J., Ana, A., Salinero, A. P., Hoppe, E. G. L., Gomes, L., Paz-Silva, A., Rebelo, M. T., \& Carvalho, L. M. (2019). Gastrointestinal parasites of free-range chickens-A worldwide issue. Bulletin of University of Agricultural Sciences and Veterinary Medicine Cluj-Napoca. Veterinary Medicine, 76(2), 110-117.

Moreira, F. L. A., Rocha, F. R. T., Coelho, K. O., Luis, R. S., Sobrinho, J. C. D., Santos, H. S. V. (2017). Comparação de técnicas para identificação de helmintos em criações de frangos caipiras no município de São Luís de Montes Belos-Go. Anais do Congresso de Ensino, Pesquisa e Extensão da UEG (CEPE).

Neves, J., Infante, S., \& Ministro, J. (2005). Impacts of very high tension power lines on birds in Portugal. In Sociedade Portuguesa para o Estudo das Aves and Quercus Associaç ão Nacional de Conservaç ão da Natureza.

Prakashbabu, B. C., Thenmozhi, V., Limon, G., Kundu, K., Kumar, S., Garg, R., Clark, E. L., Rao, A. S. R. S., Raj, D. G., Raman, M., Banerjee, P. S., Tomley, F. M., Guitian, J., \& Blake, G. P. (2017). A ocorrência de espécies de Eimeria varia entre regiões geográficas e sistemas de produção de aves e pode influenciar a diversidade genética dos parasitas. Parasitologia Veterinária, 233(15), 62-72.

Puttalakshmamma, G. C., Ananda, K. J., Prathiush, P. R., Mamatha, G. S., \& Rao, S. (2008). Prevalência de parasitas gastrointestinais de aves de capoeira e em torno de Banglore. Mundo Veterinário, 1(7), 201-202.

Quadros, R. M., Wiggers, S. B., Paes, M. P. V., \& Marques, S. M. T. (2015). Prevalência de endo e ectoparasitos de galinhas caipiras em pequenas propriedades da região serrana de Santa Catarina. PUBVET, 9(1), 1-5. https://doi.org/10.22256/pubvet.v9n1.1-5

Rennó, P. P., Queiroz, F. M., Garcia, B. P., Prado, R. N. A., Simões, M. M., Souza, J. P. F., Almeida, M. V., Souza, M. G., Bassan, L. M., \& Pereira, R. E. P. (2008). Endoparasitose em aves-revisão de literatura. Revista Científica Eletretrônica de Medicina Veterinária, 6(11), 1-6.

Siqueira, G. B. D., \& Marques, S. M. T. (2016). Parasitos intestinais em galinhas caipiras da região metropolitana de Porto Alegre, RS. PUBVET, 10(9), 690-695. https://doi.org/10.22256/pubvet.v10n9.690-695

Vicente, J. J., Rodrigues, H. O., Gomes, D. C., \& Pinto, R. M. (1995). Nematóides do Brasil. Parte IV: nematóides de aves. Revista Brasileira de Zoologia, 12(1), 1-273. https://doi.org/10.1590/s0101$\underline{81751995000500001}$

Vieira, S. (2009). Como elaborar questionários. In Como elaborar questionários (p. 159).

Vieira, F. E. G. (2010). Helmintofauna em frangos (Gallus gallus domesticus, LINNAEUS, 1758) criados em sistema colonial/caipira na região norte do Estado do Paraná [mestrado]. 2010. Universidade Estadual de Londrina, (PR).

Williams, R. B. (1999). Three enzymes newly identified from the genus Eimeria and two more newly identified from E. maxima, leading to the discovery of some aliphatic acids with activity against coccidia of the domesticated fowl. Veterinary Research Communications, 23(3), 151-163.

Willis, H. H. (1921). A simple levitation method for the detection of hookworm ova. Medical Journal of Australia, 2(18), 375-376. https://doi.org/10.5694/j.1326-5377.1921.tb60654.x

Wikipedia - Carmo do Paranaíba. (2017). Disponível em https://pt.wikipedia.org/wiki/ Carmodo Paranaíba. Acesso em 10 de junho de 2017.

Recebido: 10 de fevereiro, 2020.

Aprovado: 16 de março, 2020.

Disponível online: 14 agosto, 2020.
Licenciamento: Este artigoé publicado na modalidade Acesso Aberto sob a licença Creative Commons Atribuição 4.0 (CC-BY 4.0), a qual permite uso irrestrito, distribuição, reprodução em qualquer meio, desde que $o$ autor e a fonte sejam devidamente creditados. 